\title{
Medical Association Service Pattern of Collaborative Stroke Prevention and Treatment Based on Cloud Computing
}

\author{
Hua Gu, Lei Huang, Bei Xi and QiuLi Qin \\ School of Economics and Management \\ Beijing Jiaotong University \\ Beijing, China \\ guh@mail.bsti.ac.cn,lhuang@bjtu.edu.cn,12120664@bjtu.edu.cn, \\ qlqin@bjtu.edu.cn
}

\begin{abstract}
At present, the distribution of China's medical resources is uneven. Every day big hospitals are overwhelmed. The problem that it's difficult and expensive for ordinary people to see doctors is becoming a major social host issue. And major chronic diseases are threatening human health. In order to optimize medical resource allocation and provide more efficient services for patients with major chronic diseases, this paper takes stroke as the entry point and proposes a new medical service pattern. Namely, taking stroke patients as the center, medical association collaborates to prevent and treat them. Because cloud computing is dynamic and scalable and in order to expand to other major diseases and attract more medical associations to join when the medical service pattern is mature, this paper uses cloud computing to support the pattern. And this paper details the new medical service pattern, the framework and the service of cloud platform of medical association collaborating to prevent and treat stroke. The pattern can consider the prevention, treatment, rehabilitation, and other aspects of stroke. And it can achieve the circular medical service pattern which centered on stroke patients and can provide better service for stroke patients.
\end{abstract}

Keywords: cloud computing, stroke, medical association, collaborative prevention and treatment

\section{Introduction}

At present, China's advantage medical resources are concentrated in large metropolitan and hospitals, but the high-quality medical resources of local and community health service institutions geared to the needs of the masses are lacking. The problem that it's difficult and expensive for ordinary people to see doctors is becoming a major social host issue. For a long time, the large hospitals are overcrowded, while there are very few patients in small hospitals. It's mainly due to the unbalance of health resources allocation and service efficiency [1].In order to deepen the reform of the medical and health system and optimize the allocation of medical resources, China puts forward the concept of medical association in the twelfth five-year plan. It is composed of a tertiary hospital, secondary hospitals and community health centers within a certain area [2].The country begins to gradually promote the optimization and reorganization of regional medical resources and build medical association [3]. In this paper, the selection of medical association relies on the project "Beijing health reform of the medical service pattern and demonstrations of medical association collaborating to prevent and treat major chronic diseases" The medical association is composed of Beijing Tiantan Hospital (a tertiary hospital, BTTH), Beijing Puren Hospital (a secondary hospital, BPRH), and Beijing Fangzhuang Community Health Service Center (a community, BFZC). BTTH is a neurology-focused hospital. According to the results of the third time of the National 
Health Ministry's death investigation, stroke, one of the major chronic diseases, is the first cause of death [4]. Stroke, also called Cerebral Apoplexy, is a clinical event of localized or diffused impairment of brain function, quickly caused by acute damage to the cerebral circulation. It is a common disease in the elderly with high incidence and disability rate, which may cause heavy financial and emotional burden to the society and family [5]. Therefore, the prevention of stroke is of great social significance.

In order to optimize medical resource allocation and provide more efficient services for patients with major chronic diseases, this paper relying on the project proposes a new medical service pattern. It's a medical service pattern of medical association collaborating to prevent and treat major chronic diseases. However, the information among the health care institutions in the medical association is isolated. In order to realize the sharing of medical resources for the health care institutions in the medical association, expand to other major diseases and attract more medical associations to join when the medical service pattern is mature, we must use a technology. Cloud computing is a computing mode which provides dynamic, virtualized resources through the Internet as a service. Cloud computing is high of scalability and can provide flexible service, so it can help break the information isolated island problem between medical institutions. Cloud computing can integrate existing healthcare information systems and connect information islands to build a large-scale resource pool, and ultimately realize the sharing of medical information resources [6].Thus, this paper proposes medical association service pattern of collaborative stroke prevention and treatment based on cloud computing.

In this paper, section 1 analyzes the current situation of medical association, including BTTH, BPRH, BFZC, and proposes medical association service pattern of collaborative stroke prevention and treatment. Section 2 puts forward the framework of cloud platform. Then, the services of cloud platform are described in detail in section 3 . Finally, the paper makes a summary.

\section{Stroke Medical Association Service Pattern}

\section{A. Current Situation of Stroke Medical Association}

In this paper, the selection of medical association relies on the project. It's composed of BTTH, BPRH and BFZC. Currently, the information construction of BFZC is first-class, and it can establish a comprehensive health records for stroke patients who first diagnosed in BFZC. What's more, it can screening for high-risk groups. But, it can't get information of stroke patients in other hospitals. BPRH can also create electronic medical records and other information for stroke patients coming to BPRH, but it can't get information of stroke patients in other hospitals. Similarly, the information construction of BTTH is quite superb, and it can create detailed electronic medical records for stroke patients diagnosed in BTTH. But it can't obtain stroke patients information in other hospitals, including BFZC, BPRH. Doctors can only make stroke patients re-examination, inspection, which may delay the best treatment time. Also, there are too many stroke patients in BTTH every day. In fact, many stroke patients with less severe disease may go to BFZC or BPRH to treat. And some stroke patients with severe may not get the chance to see a doctor and delay be treated. In addition, the beds are limited in BTTH and maybe occupied by many rehabilitation stroke patients, it cannot accept more new stroke patients. It can be seen that stroke patients' information in the three hospitals are not shared and there is no good cooperation way for the three hospitals to provide services for stroke patients.

\section{B.Medical Association Collaborative Stroke Prevention and Treatment Service}

\section{Pattern}

In order to alleviate the difficult and expensive medical treatment problems and provide better services for stroke patients, this paper proposes a new medical pattern of medical association collaborating to prevent and treat stroke. The new pattern is shown in Figure1.

The new medical service pattern is centered in stroke patients. Community health centers do primary prevention for normal populations and can screen high-risk groups. 
Emergency centers do emergency management for stroke patients. Tertiary hospitals and secondary hospitals treat stroke patients and then rehabilitation centers provide rehabilitation treatment for stroke patients. After discharge, the community health centers do secondary prevention for stroke patients. Namely, the pattern is centered in stroke patients to achieve the primary prevention of community health service centers, emergency response of emergency centers, upward referral to the tertiary hospital or secondary hospital, downward referral to the secondary hospital or community health service center from stroke prevention, treatment, rehabilitation, and other aspects.

The responsibilities of the three hospitals in the medical association are distributed as follows.

BFZC provides first diagnosis service for residents of the community, screens stroke high-risk groups from the normal population and does health education for them. In addition, according to the stroke patient's condition, they can be transferred to BTTH or $\mathrm{BPRH}$. And it does rehabilitation treatment for stroke patients in rehabilitation phase transferred from BTTH or BPRH.

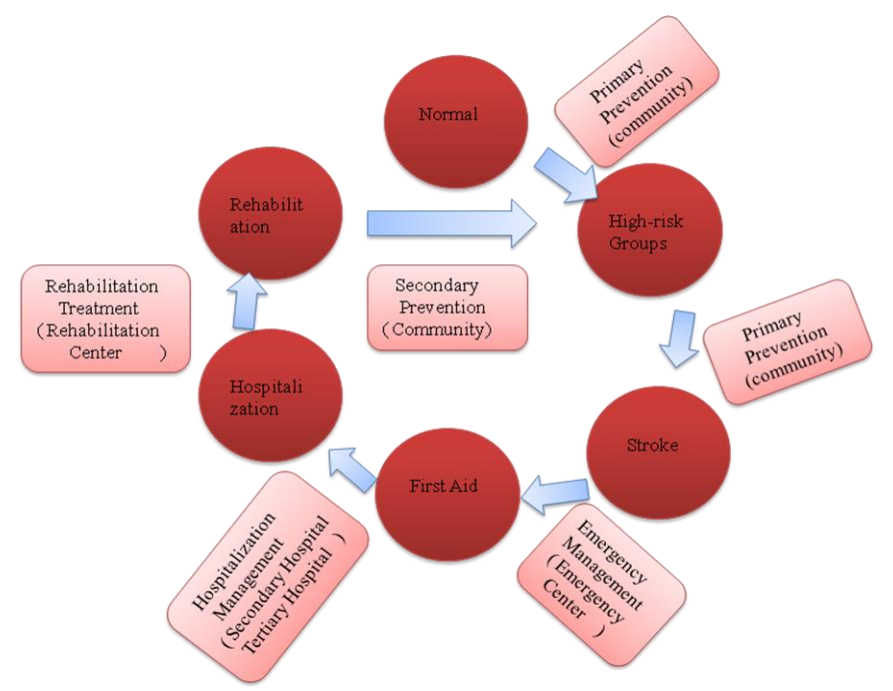

Figure 1. Stroke Medical Association Service Pattern

BPRH can do further assessment for stroke patients. According to the hospital medical level, BPRH can treat stroke patients who are not serious and transfer serious stroke patients to BTTH. Also, BPRH researches the rehabilitation technical specifications and does rehabilitation treatment for stroke patients in rehabilitation phase.

BTTH can focus on the study of complex diseases and determine clinical pathways, key indicators for stroke patients. It can treat serious stroke patients and transfer them to BPRH or BFZC when they are in the rehabilitation phase.

In addition, if one of the hospitals does not need to transfer stroke patients, it needs other hospitals for collaborative consultation. It may request other hospitals for collaborative consultation. And they must share the medical information of stroke patients.

Cloud computing is a computing mode which provides dynamic, virtualized resources through the Internet as a service. It can help break the information isolated island problem between medical institutions. And it can provide an effective, low-cost solution to achieve this service pattern. So the medical service pattern of medical association collaborating to prevent and treat stroke based on cloud computing will be detailed in the following.

\section{The Framework of Stroke Medical Association Cloud Platform}

In order to realize the new medical association medical service pattern, we need build a platform that is built on top of the existing systems of medical association to realize the 
medical information sharing of stroke patients, smooth two-way referral and collaborative consultation. The paper uses the cloud computing technology to build the cloud platform. The framework of cloud platform is divided in three layers [7]. It's shown in Figure 2.

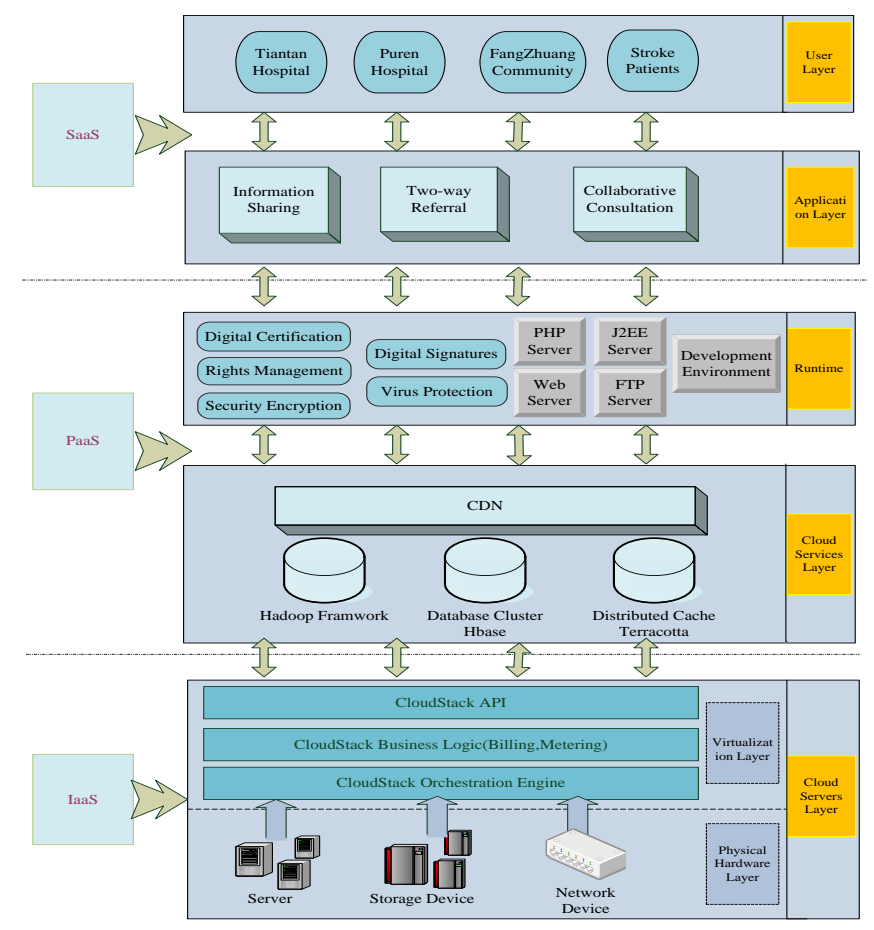

Figure 2. The Framework Of Stroke Medical Association Cloud Platform

\section{IaaS layer (Infrastructure-as-a-Service)}

IaaS layer is cloud server's layer divided into two layers, including physical hardware layer and virtualization layer. Physical hardware layer has servers, storage devices and network devices. We select PC servers installed the operating system of Linux centos version with virtualization management, network storage NFS and network equipment including routers, switches. The physical layer virtualized into multiple virtual servers by virtualization layer using cloudstack technology. CloudStack is cloud platform management software. It allows deploying and managing multi-tenant private clouds with large scale virtual machines. It also supports a number of popular hypervisors such as KVM, VMware, and XenServer. CloudStack can accelerate highly scalable public and private cloud deployment, management and configuration. Using CloudStack as the foundation, data center operators can quickly and easily create cloud services through existing infrastructure [8]. CloudStack and KVM nicely support the parallel live migration of multiple virtual machines. The platform can provide flexible services and expand easily. After the pattern runs mature, it can be expanded to other major diseases and add more medical associations.

D. PaaS layer (Platform-as-a-Service)

PaaS layer packets the underlying virtual resources into services and can provide interfaces so that other systems can access the cloud platform. PaaS layer includes cloud services layer and runtime. IaaS virtualizes the servers into multiple virtual machines. According to the need, the virtual machines were installed different operating systems, such as windows server2008, centos6.4 and so on.

The virtual machines installed centos operating systems are required to install the following services. 
- Hadoop Distributed Framework: The cores are distributed file system HDFS (Hadoop Distributed FileSystem) and distributed computing MapReduce. And it can provide underlying distributed infrastructure for users.

- Database Cluster Hbase: Hbase is a high-reliability, high performance, column-oriented, scalable, distributed storage system. In addition, according to need, the database cluster can also select the relational database, like MySQL, or select non-relational database, like MongoDB, Redis, etc.

- Distributed Cache Terracotta: It can realize the high performance, high availability and high stability of the enterprise java cluster. It can also alleviate the bottlenecks between the java clustering and the database cluster.

The virtual machines installed windows server 2008 operating systems are required to install the following services.

- Development Environment: The Visual Studio, Eclipse can be installed. They can provide development environment for developers.

- Application Server: According to the need, PHP Server, Web Servers, J2EE Servers, FTP servers can be installed.

- System Applications: The system applications can be deployed on the virtual machine.

Since the security of stroke patient information is very important, we use some methods to ensure the security [9]. For example, the digital certification (X.509V3 format), digital signatures (including the original data, electronic signatures, trusted time), security encryption (SSL), rights management (control technology RBAC), virus protection, and so on. CDN (Content Distribution Network) is that it copies the data of cloud platform to the server closer to the hospitals. The CDN is optional. Hospitals can faster get the relevant data.

\section{- SaaS layer (Software-as-a-Service)}

SaaS layer is composed of application layer and user layer. Every application is provided in the form of cloud service. Applications include information sharing system, two-way referral system, and collaborative consultation system. These systems are deployed on the virtual machines installed windows server 2008 and provide services to the user through web services of the virtual machines. User layer includes BTTH, BPRH, BFZC and stroke patients who agree to store their information to the cloud platform. Users can access the systems through the browser. The following will detail the cloud platform services.

\section{The Services of Stroke Medical Association Cloud Platform}

The cloud platform is built on top of the existing systems of BTTH, BPRH and BFZC. The services of the cloud platform are shown in Figure 3.

In order to realize the new medical service pattern, the cloud platform provides three services, including information sharing, two-way referral, and collaborative consultation. Information sharing is the basic service for the other two services.

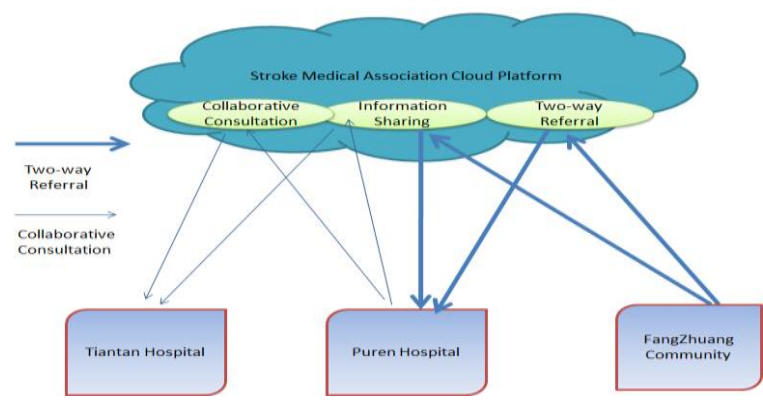

Figure 3. The Services Of Stroke Medical Association Cloud Platform 
The bold lines represent two-way referral. For example, BFZC transfers stroke patient to the BPRH, then BFZC calls the two-way referral service and upload the information of stroke patients to the cloud platform. At the same time, BPRH can receive the referral information and view information of the stroke patient from the cloud platform.

The fine lines represent collaborative consultation. For example, BPRH need BTTH collaborative consultation, and then BPRH calls the collaborative consultation service and upload the information of stroke patients to the cloud platform. At the same time, BTTH can receive the collaborative consultation information and view information of the stroke patient from the cloud platform.

The detailed various modules of the cloud platform services will be introduced below.

\section{- Cloud Platform Information Sharing}

When the medical association needs referral or collaborative consultation, they need to call the information sharing system which stores the stroke patients' data. It is composed of upload data module and query data module.

1) Upload Data

If the medical association needs to share data of stroke patients in their own hospitals to the cloud platform, they need to call the upload data module of information sharing system. Upload data flow diagram of cloud platform is shown in Figure4.

a) First, doctors in the medical association login the cloud platform of medical association collaborating to prevent and treat stroke through the browser and enter the upload data module of information sharing system.

b) According to the condition of stroke patients, doctors click the select data button and choose stroke patients' data which are needed to be uploaded.

c) Doctors click the data conversion button. Because the data standards of hospitals in the medical association are different, the HL7 engine in the information sharing system of cloud platform will convert the data into a cloud platform standard data through HL7 message structure

d) Doctors click the upload data button. After receiving the data, $\mathrm{HL} 7$ engine will do HL7 message parsing for the data. Then the data will be stored to the databases in cloud platform data standard.

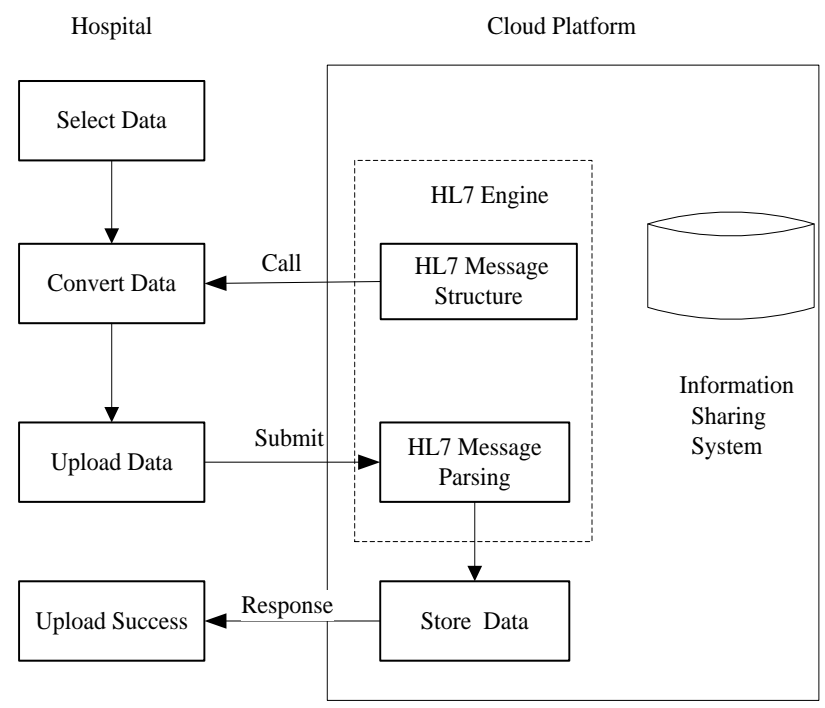

Figure 4. Upload Data Of Information Sharing Service 


\section{2) Query Data}

If the medical association needs to query data of stroke patients in the cloud platform, they need to call the query data module of information sharing system. Query data flow diagram of cloud platform is shown in Figure5.

\section{Hospital}

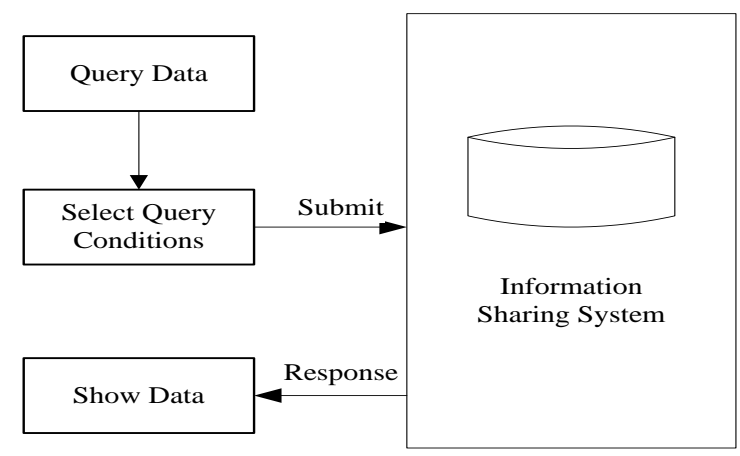

Figure 5. Query Data Of Information Sharing Service

a) First, doctors in the medical association login the cloud platform of medical association collaborating to prevent and treat stroke through the browser and enter the query data module of information sharing system.

b) According to the need, doctors click the select query condition button, select query conditions and submit the conditions to the cloud platform.

c) The query data module will complete the data query in the background according to the user's query conditions.

d) The cloud platform will display the data of stroke patients on the users' interface.

- Cloud Platform Two-Way Referral

If medical association needs transfer stroke patients, they need to call the two-way referral system. Two-way referral flow diagram of cloud platform is shown in Figure6.

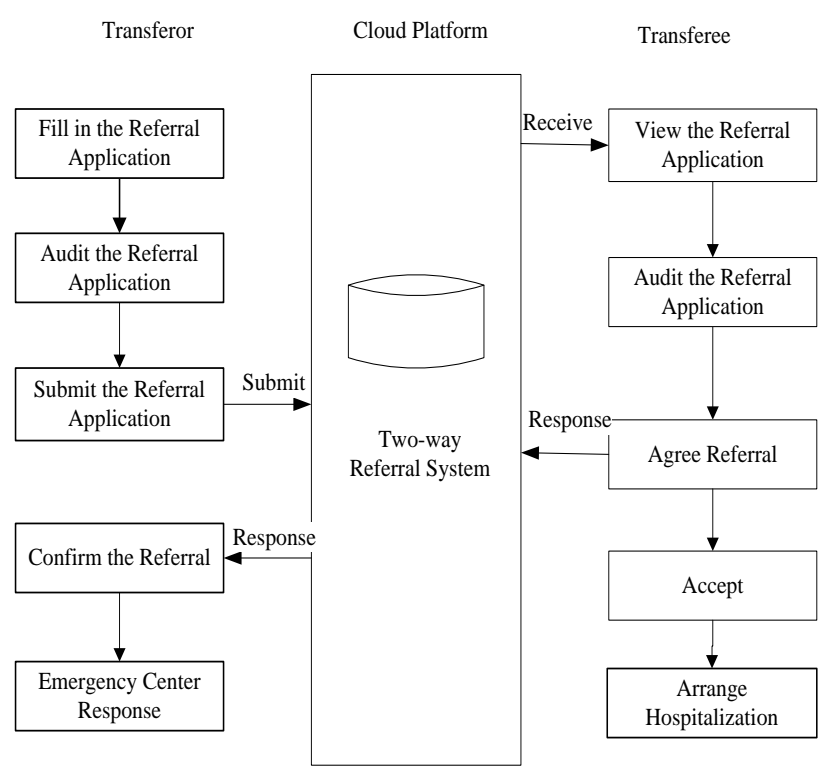

Figure 6. Cloud Platform Two-Way Referral Service 
Transferor is that the hospital transfers its stroke patients to other hospitals.

Transferee is that the hospital accepts the stroke patients from other hospitals.

e) The doctors of transferor login the cloud platform through the browser and call the two-way referral system.

f) The doctors of transferor fill in the referral application according to the patients' condition.

g) The audit staff of transferor audits the referral application.

h) The doctors of transferor submit the audited referral application to the cloud platform.

i) The cloud platform will send the received referral application to the transferee.

j) The doctors of transferee view the referral application and audit it.

k) The doctors of transferee send agree referral message to the cloud platform.

l) The cloud platform sends agree referral message to the transferor.

$m$ ) The doctors of transferor confirm the referral and arrange emergency center to transfer stroke patients. At the same time, the doctors of transferee ready to receive the stroke patients and arrange hospitalization.

\section{- Cloud Platform Collaborative Consultation}

If one of the hospitals in the medical association doesn't need to transfer stroke patients and needs other hospitals' collaborative consultation, it can call the collaborative consultation system. Collaborative consultation flow diagram of cloud platform is shown in Figure7.

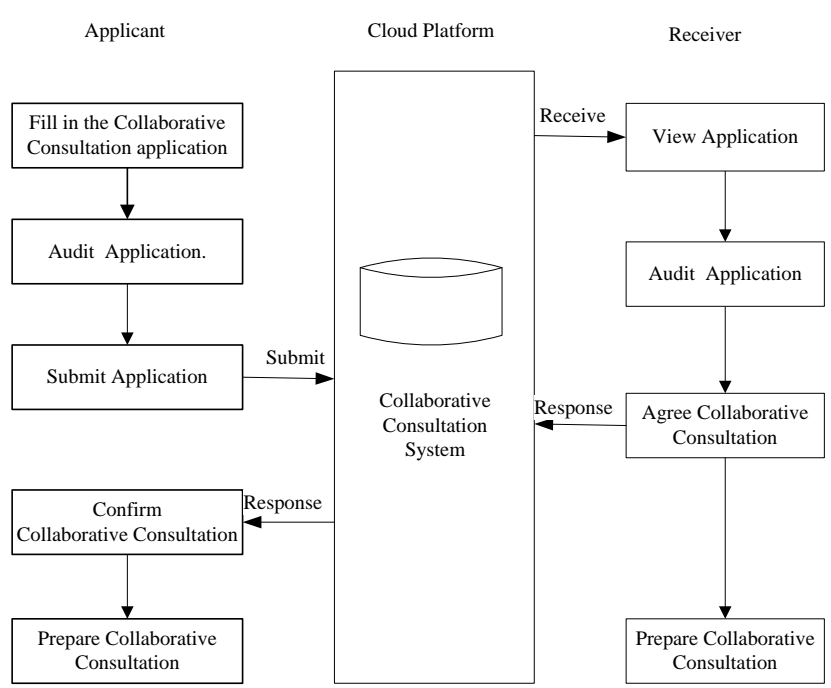

\section{Figure 7. Cloud Platform Collaborative Consultation Service}

Applicant is that the hospital needs other hospitals' collaborative consultation.

Receiver is that the hospital accepts the collaborative consultation.

n) The doctors of applicant login the cloud platform through the browser and call the collaborative consultation system.

o) The doctors of applicant fill in the collaborative consultation application according to the patients' condition.

p) The audit staff of applicant audits the collaborative consultation application.

q) The doctors of applicant submit the audited collaborative consultation application to the cloud platform. 
r) The cloud platform will send the received collaborative consultation application to the receivers.

s) The doctors of receiver query the collaborative consultation application and audit it.

t) The doctors of receiver send agree consultation application message to the cloud platform.

u) The cloud platform sends agree consultation application message to the applicant.

v) Applicant and receiver ready to do collaborative consultation. For example, they arrange remote meeting rooms and arrange the responsible doctors and so on.

\section{Summary}

The current uneven distribution of medical resources results in difficult and expensive medical treatment problems. Based on the analysis of the current situation of stroke patient services in the medical association composed of BTTH, BPRH, and BFZC, this paper proposes medical service pattern of medical association collaborating to prevent and treat stroke. The pattern is stroke patient-oriented and undertakes stroke prevention, treatment, rehabilitation, and other aspects in order to achieve circular medical service pattern. Implementation of this service model requires technical supports. This paper also uses cloud computing technology to build a cloud platform that is built on top of the existing systems of medical association. The framework of the cloud platform and the services that the cloud platform provides are introduced in detail. The cloud platform can provide the medical information sharing of stroke patients and ensure smooth two-way referral, collaborative consultation between the hospitals in the medical association. The medical service pattern of medical association collaborating to prevent and treat stroke based on cloud computing can provide better services for stroke patients. It also can provide a reference for other major diseases and medical association service pattern.

\section{Acknowledgment}

The authors would like to express their great thanks to the support from the Beijing municipal science \& technology commission Grant No Z131100005613017.

\section{References}

[1] Y. Luan, "Research on the trend and situation in integration of medical resources", Medicine \& Philosophy, Humanistic \& Social Medicine Edition, vol. 1, (2014), pp. 65-67.

[2] L. Zhang and M. Gu, "Development Strategy on Regional Medical Association", Chinese Health Quality Management, vol. 21, (2014), pp.74-76.

[3] W. J. Ren, "Exploration on the Problems and Countermeasure of the Cultural Integration in Medical Association in China", Medicine and Society, vol. 27, (2014), pp. 31-34.

[4] Chinese Ministry of Health, "The Main Case of the Third National Investigation of Death Causes", Bulletin of Chinese Cancer, vol. 17, (2008), pp. 344-345.

[5] Y. F. Wei and W. J .Zhao, "Causes \& Risk factors of Young Adult Stroke", Medical Recapitulate, vo1. A14, (2008) August, pp. 2315-2317.

[6] L. Z. Kong, "Cloud Computing Application in Medical and Health Information", Journal of Shanxi Medical College For Continuing Education, vol. 22, (2012), pp. 69-70.

[7] B. Xi, Q. L Qin and L. Huang, "The Regional Medical Business Process Optimization Based on Cloud Computing Medical Resources Sharing Environment", Cybernetics and Information Technologies, vol. 13 (Special Issue), (2013), pp. 18-29.

[8] http://cloudstack.apache.org/

[9] Y. Y. Guo, M. H. Kuo and T. Sahama, "Cloud computing for healthcare research information sharing", Proceedings of the 2012 IEEE 4th International Conference on Cloud Computing Technology and Science (CloudCom), IEEE Computer Society, (2012) December, pp. 889-894. 
International Journal of Grid Distribution Computing Vol. 8, No.4, (2015) 